\title{
Efficient Ultrasound Synthesis, Characterizations and Antimicrobial Screening of Novel Cyclic $\beta$-Diketones
}

\author{
N. S. KORDE ${ }^{\mathrm{a}}$, S. T. GAIKWAD ${ }^{\mathrm{b}}$, B. C. $\mathrm{KHADE}^{\mathrm{c}}$ and A. S. RAJBHOJ ${ }^{\mathrm{b}}$ \\ ${ }^{a}$ Dayanand Science College, Latur -413512, (M.S.), India \\ bepartment of Chemistry, Dr. Babasaheb Ambedkar Marathwada University, \\ Aurangabad (M.S.), 431004, India \\ ${ }^{\mathrm{c}}$ D. S. M. College, Parbhani (M.S.), India \\ nandineekorde0@gmail.com
}

Received 17 August 2012 / Accepted 19 September 2012

\begin{abstract}
Hydroxyphenyl)-3-propane,1,3-diones $\mathbf{4}\left(\mathbf{L}_{\mathrm{A}}-\mathbf{L}_{\mathrm{F}}\right)$ have been synthesized by a simple and convenient method employing Baker-Venkatraman transformation on corresponding 2acetylphenyl benzoate by conventional as well as ultrasound irradiation method. The structure of synthesized compounds has been assigned on the basis of elemental and spectral analysis (IR, ${ }^{1} \mathrm{H}$ NMR, ${ }^{13} \mathrm{C}$ NMR, UV/Vis, Mass). The synthesized compounds were evaluated for antibacterial and antifungal activities. Utilization of ultrasound irradiation, simple reaction conditions, isolation and purification makes this manipulation very interesting from an economic and environmental perspective.
\end{abstract}

Keywords: 2-Acetylphenyl benzoate, Cyclic $\beta$-diketones, Baker-Venkataraman rearrangement, Antimicrobial screening, Ultrasound irradiation

\section{Introduction}

Cyclic $\beta$-diketones have gained a lot of interest due to their importance as good ligands ${ }^{1,2}$ for the chelation with metals, as intermediate in the synthesis of core heterocycles such as flavones $^{3}$, benzodiazepine ${ }^{4}$, pyrazole ${ }^{5}$, isoxazole ${ }^{6}$ and pyrimidine ${ }^{7}$ and triazole ${ }^{8}$. $\beta$-Diketones have been pharmacological activities like prophylactic antitumor ${ }^{9}$, antiviral ${ }^{10}$, antibacterial ${ }^{11}$, systematic insecticidal ${ }^{12}$ and antioxidant ${ }^{13}$. It has been used as an anti-sunscreen agent ${ }^{14}$. $\beta$-diketones are well known to have keto-enol tautomerism ${ }^{15}$ and recently it is reported that they have the important pharmacophores for the HIV-integrase $(1 \mathrm{~N})$ inhibitors ${ }^{16}$. Further, it has been reported recently that a number of $\beta$-diketones has warrant examination as breast cancer chemopreventative blocking agent ${ }^{17}$, anticarcinogenic agent ${ }^{18}$ and antiestrogenic agent $^{19}$.

Owing to $\beta$-diketones having such varying pharmacological activities, we became interested to synthesize a series of novel $\beta$-diketones. However, in most cases, synthesis of 
$\beta$-diketone by ultrasound irradiation method has received less attention. With this view here we report the synthesis of novel cyclic $\beta$-diketones $\mathbf{4}\left(\mathbf{L}_{\mathbf{A}}-\mathbf{L}_{\mathbf{F}}\right)$ under ultrasound irradiation using Baker-Venkatraman rearrangement and the synthesized compounds were evaluated for antibacterial and antifungal screening.

Ultrasound irradiation assisted organic synthesis is an efficient and eco-friendly synthetic strategy. Many homogeneous and heterogeneous reactions can be conducted smoothly by sonication to provide improved yields and increased selectivities ${ }^{20}$. Therefore ultrasound irradiation has been established as an important technique in organic synthesis.

\section{Experimental}

2-Hydroxy acetophenone 1(A) was prepared by Fries migration of phenyl acetate using anhydrous $\mathrm{AlCl}_{3}$. All the solvents and reagents used were of synthetic grade.

\section{Measurements}

Melting points were determined in open glass capillaries and were uncorrected. ${ }^{1} \mathrm{H}$ NMR and

${ }^{13} \mathrm{C}$ NMR spectra were recorded on a Varian -NMR-mercury 300 using tetra methyl silane as an internal standard and $\mathrm{CDCl}_{3}$ as solvent. FT-IR spectra were recorded using $(\mathrm{KBr})$ disc on Bruker spectro-photometer. Mass spectra were taken on a Macro mass spectrometer. UV/Vis spectra were recorded on UV/Vis spectrophotometer model UV-1601, SHIMADZU, Japan. Elemental analyses were done using Perkin Elmer 2400CHN analyzer.

\section{Preparation of 2-acetylphenyl benzoate (3A-F)}

To the mixture of $o$-hydroxyacetophenone $(1.36 \mathrm{~g}, 0.01 \mathrm{~mol})$ and 4-methoxy benzoic acid $(1.52 \mathrm{~g}, 0.01 \mathrm{~mol})$ a dry pyridine $(5 \mathrm{~mL})$ and $\mathrm{POCl}_{3}(1 \mathrm{~mL})$ were added drop wise with constant stirring at $0{ }^{\circ} \mathrm{C}$. The reaction mixture was irradiated for about $3-4 \mathrm{~h}$ under ultrasound. After completion of the reaction (monitored by TLC), the reaction mixture was poured into $100 \mathrm{~mL} \mathrm{HCl}(1 \mathrm{M})$ containing $50 \mathrm{~g}$ of crushed ice and solid obtained was filtered and washed with $10 \mathrm{~mL}$ ice-cold methanol and then with $10 \mathrm{~mL}$ of water. It was recrystallized from ethanol, filtered and dried.

\section{Preparation of 2-hydroxyphenyl-3-phenylpropane-1,3diones $\mathbf{4}\left(\mathbf{L}_{A}-\boldsymbol{L}_{\boldsymbol{F}}\right)$}

Compound $3 \mathbf{A}$ ( $2.7 \mathrm{~g}, 0.01 \mathrm{~mol}$ ) was dissolved in dry pyridine $(10 \mathrm{~mL})$. To this powdered $\mathrm{KOH}$ (1.12 g, $0.02 \mathrm{~mol}$ ) was added and the reaction mixture was irradiated for about 2-3 h under ultrasound. After completion of reaction (monitored by TLC), the reaction mixture was poured into ice cold water and acidified with conc. HCl. The yellow solid obtained was filtered off and crystallized from absolute ethanol to obtain pure product. The analytical data of the compounds $\mathbf{4}\left(\mathbf{L}_{\mathrm{A}}-\mathbf{L}_{\mathbf{F}}\right)$ are given in Table 1. The spectral data for various $\mathbf{4}\left(\mathbf{L}_{\mathrm{A}}-\mathbf{L}_{\mathrm{F}}\right)$ are described below.

Table 1. The analytical data of compounds $4\left(\mathbf{L}_{\mathrm{A}}-\mathbf{L}_{\mathrm{F}}\right)$

\begin{tabular}{|c|c|c|c|c|c|c|}
\hline \multirow{3}{*}{ Compd. } & \multirow{3}{*}{$\begin{array}{l}\text { Molecular } \\
\text { Formula }\end{array}$} & \multirow{3}{*}{ Mol. Wt. } & \multicolumn{4}{|c|}{$\%$ Analysis } \\
\hline & & & \multicolumn{2}{|c|}{$\% \mathrm{C}$} & \multicolumn{2}{|c|}{$\% \mathrm{H}$} \\
\hline & & & Cald & Found & Cald & Found \\
\hline $4 L_{A}$ & $\mathrm{C}_{16} \mathrm{H}_{14} \mathrm{O}_{4}$ & 270 & 71.10 & 71.01 & 5.22 & 5.13 \\
\hline $4 \mathrm{~L}_{\mathrm{B}}$ & $\mathrm{C}_{17} \mathrm{H}_{16} \mathrm{O}_{4}$ & 284 & 71.82 & 71.10 & 5.67 & 5.52 \\
\hline $4 L_{C}$ & $\mathrm{C}_{15} \mathrm{H}_{11} \mathrm{O}_{3} \mathrm{Br}$ & 319 & 56.45 & 56.09 & 3.47 & 3.20 \\
\hline $4 L_{D}$ & $\mathrm{C}_{16} \mathrm{H}_{13} \mathrm{O}_{4} \mathrm{Cl}$ & 304.65 & 63.06 & 62.98 & 4.30 & 4.11 \\
\hline $4 \mathrm{~L}_{\mathrm{E}}$ & $\mathrm{C}_{17} \mathrm{H}_{15} \mathrm{O}_{4} \mathrm{Cl}$ & 318.5 & 64.06 & 63.93 & 4.74 & 4.60 \\
\hline $4 L_{F}$ & $\mathrm{C}_{15} \mathrm{H}_{10} \mathrm{O}_{3} \mathrm{ClBr}$ & 353.5 & 50.95 & 50.81 & 2.85 & 2.35 \\
\hline
\end{tabular}


4L $\mathrm{L}_{\mathrm{A}}$ : FT-IR $(\mathrm{KBr}) \mathrm{cm}^{-1}: 2912.98(-\mathrm{OH}), 1708.01(\mathrm{C}=\mathrm{O}), 1487.74(\mathrm{Ar} \mathrm{C}=\mathrm{C}) .{ }^{1} \mathrm{H}-\mathrm{NMR}$ $\left(300 \mathrm{MHz}, \mathrm{CDCI}_{3},-\mathrm{d} 6\right): \delta=7.9(\mathrm{~d}, 3 \mathrm{H}, \mathrm{Ar}-\mathrm{H}), 6.8(\mathrm{~m}, 5 \mathrm{H}, \mathrm{Ar}-\mathrm{H}), 7.4(\mathrm{q}, 1 \mathrm{H},=\mathrm{CH}-), 3.9$ $\left(\mathrm{s}, 3 \mathrm{H}, \mathrm{OCH}_{3}\right), 12.2$ (s, $\left.1 \mathrm{H}, \mathrm{OH}\right), 15.9$ (s, $1 \mathrm{H}$, Enolic-OH), ${ }^{13} \mathrm{C}-\mathrm{NMR}\left(300 \mathrm{MHz}, \mathrm{CDCl}_{3}\right), \delta$ 190.0(s, C-1,C=O), 92.8 (s, C-2,-CH=), 185.1(S,C-3), 126.0(d, C-1',C-1'”), 162.8 (s,C-2'), 118.4 (s,C-3'), 135.8 (s,C-4'), 119.3 (s,C-5'), 128.7 (s, C-6,'), 128.0 (d, C-2', C-6'”), 114.1(d, C-3', C-5'”), 162.0 (s, C-4'”), 55.8 (s,C-7', $0 \mathrm{CH}_{3}$ ). UV/Vis (DMSO)nm: 370,410; EC-MS :270.28 (M+23).

4L L $_{\mathbf{B}}$ FT-IR (KBr) cm ${ }^{-1}: 2921.31(-\mathrm{OH}), 1741.68(\mathrm{C}=\mathrm{O}), 1486.11(\mathrm{Ar} \mathrm{C}=\mathrm{C}) .{ }^{1} \mathrm{H}-\mathrm{NMR}$ (300 MHz, $\left.\mathrm{CDCl}_{3}-\mathrm{d} 6\right) ; \delta=7.85(\mathrm{~d}, 3 \mathrm{H}, \mathrm{Ar}-\mathrm{H}), 6.85(\mathrm{~m}, 5 \mathrm{H}, \mathrm{Ar}-\mathrm{H}), 7.4(\mathrm{q}, 1 \mathrm{H},=\mathrm{CH}-), 4.1$ $\left(\mathrm{q}, 2 \mathrm{H},-\mathrm{OCH}_{2}\right), 1.7$ (t,3H,-CH$), 12.1(\mathrm{~s}, 1 \mathrm{H}, \mathrm{OH}), 15.9$ (s, $1 \mathrm{H}$, Enolic-OH), ${ }^{13} \mathrm{C}-\mathrm{NMR}$ $\left(300 \mathrm{MHz}, \mathrm{CDCl}_{3}\right), \delta$ 189.5(s, C-1, C=O), 93.1(s,C-2, -CH=), 185.0(s,C-3), 124.5(s,C-1'), 162.5(s, C-2'), 118.0(s,C-3'), 136.0(s,C-4'), 118.5(s, C-5'), 127.5(s,C-6'), 126.0(s,C-1'”),

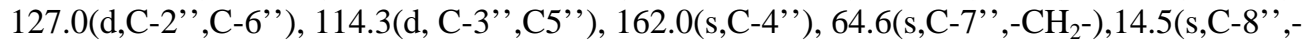
$\mathrm{CH}_{3}$ ),UV/Vis(DMSO)nm:360,410 EC-MS: $284.31(\mathrm{M}+23)$.

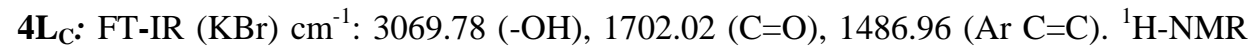
(300 MHz, $\left.\mathrm{CDCl}_{3}-\mathrm{d}_{6}\right) ; \delta=6.8(\mathrm{~d}, 3 \mathrm{H}, \mathrm{Ar}-\mathrm{H}$ ), $7.7(\mathrm{~m}, \mathrm{Ar}-\mathrm{H}), 7.39(\mathrm{~s}, 1 \mathrm{H},=\mathrm{CH}-), 12.1(\mathrm{~s}, 1 \mathrm{H}$, $\mathrm{OH}), 15.5$ (s, 1H, Enolic-OH), 13C-NMR(300 MHz, CDCl 3 ) , $190.0(\mathrm{~s}, \mathrm{C}-1, \mathrm{C}=\mathrm{O}), 93(\mathrm{~s}, \mathrm{C}-$ 2,-CH=), 184.2(s,C-3), 126.5(s, C-1'), 162.0(s,C-2'), 119.0(s,C-3')136(s,C-4'), 119.5(s,C-5'), 131.3(s,C-6'),129.4(s,C-1'’),128.6(d,C-2'”,C-6'”),131.6(d,C-3',,C5'”),122.3(s,C-4'’).

UV/Vis(DMSO)nm:360,412; EC-MS: 319.15 (M+23).

4L L $_{\mathbf{D}}$ FT-IR (KBr) cm ${ }^{-1}:$ 2919.67(-OH), $1743.31(\mathrm{C}=\mathrm{O}), 1465.02(\mathrm{Ar} \mathrm{C}=\mathrm{C}) .{ }^{1} \mathrm{H}$ NMR $\left(300 \mathrm{MHz}, \mathrm{CDCI}_{3}-\mathrm{d}_{6}\right) ; \delta=7.0(\mathrm{t}, 3 \mathrm{H}, \mathrm{Ar}-\mathrm{H}), 7.99(\mathrm{~d}, 2 \mathrm{H}, \mathrm{Ar}-\mathrm{H}), 7.85$ (s, $\left.1 \mathrm{H}, \mathrm{Ar}-\mathrm{H}\right), 7.31$ $(\mathrm{m}, 1 \mathrm{H}, \mathrm{Ar}-\mathrm{H}), 6.9$ (q, 1H,=CH-), $3.9\left(\mathrm{~s}, 3 \mathrm{H},-\mathrm{CCH}_{3}\right), 12.1$ (s, 1H,OH), 17.75 (s,1H,Enolic $\mathrm{OH}),{ }^{13} \mathrm{C}-\mathrm{NMR}\left(300 \mathrm{MHz}, \mathrm{CDCl}_{3}\right) ; \delta$ 192.5(s,C-1,C=O). 91.2(s,C-2,-CH=), 179(s,C-3), 124.5 (s,C-1'), 161.0(d,C-2',C-4' '), 120.0(s,C-3'), 134.9(s,C-4'), 128.4(t,C-5',C-2','C-6' '), 131.5

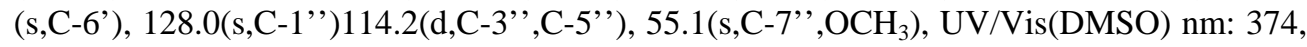
412; EC-MS : $304.73(\mathrm{M}+23)$.

4LE: FT-IR (KBr) cm ${ }^{-1}: 2977.54(-\mathrm{OH}), 1718.10(\mathrm{C}=\mathrm{O}), 1471.16$ (Ar C=C). ${ }^{1} \mathrm{H}-\mathrm{NMR}$ $\left(300 \mathrm{MHz}, \mathrm{CDCI}_{3}-\mathrm{d}_{6}\right) ; \delta=7.89(\mathrm{q}, 4 \mathrm{H}, \mathrm{Ar}-\mathrm{H}), 7.05(\mathrm{~m}, 3 \mathrm{H}, \mathrm{Ar}-\mathrm{H}), 6.61(\mathrm{~s}, 2 \mathrm{H},=\mathrm{CH}-)$, 4.1(t,2H,-OCH2),1.45 (q, 3H,-CH3),12.1 (s,1H,OH), 15.7 (s,1H, Enolic-OH), ${ }^{13} \mathrm{C}-\mathrm{NMR}$ (300MHZ, $\left.\mathrm{CDCl}_{3}\right), \delta 193.8(\mathrm{~s}, \mathrm{C}-1, \mathrm{C}=\mathrm{O}), \quad 91.2(\mathrm{~s}, \mathrm{C}-2,-\mathrm{CH}=), 179.0(\mathrm{~s}, \mathrm{C}-3), \quad 124\left(\mathrm{~s}, \mathrm{C}-1^{\prime}\right)$, 163.6(s,C-2'), 120(s,C-3'), 135.9(s,C-4,), 128(s,C-5'), 131.9(s,C-6'), 121.5(s,C-1'”), 129.5

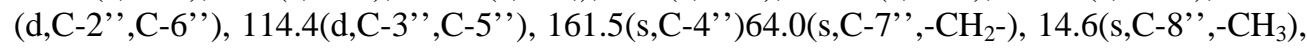
UV/Vis (DMSO)nm:360,410; EC-MS : 318.75 (M+23).

4LF: FT-IR (KBr) $\mathrm{cm}^{-1} 2916.32(-\mathrm{OH}), 1720.28(\mathrm{C}=\mathrm{O}), 1469.22(\mathrm{Ar} \mathrm{C}=\mathrm{C}) .{ }^{1} \mathrm{H}-$ NMR (300MHz, CDCl $\left.3-\mathrm{d}_{6}\right) ; \delta=7.8(\mathrm{~m}, 5 \mathrm{H}, \mathrm{Ar}-\mathrm{H}), 7.35$ (q, 1H, Ar-H), $7.01(\mathrm{~m}, 1 \mathrm{H}, \mathrm{Ar}-$ $\mathrm{H}), 6.65(\mathrm{~s}, 1 \mathrm{H},=\mathrm{CH}-), 11.95(\mathrm{~s}, 1 \mathrm{H}, \mathrm{OH}), 15.45\left(\mathrm{~s}, 1 \mathrm{H}\right.$, Enolic-OH), ${ }^{13} \mathrm{C}-\mathrm{NMR}(300 \mathrm{MHz}$, $\left.\mathrm{CDCl}_{3}\right), \delta 192.5(\mathrm{~s}, \mathrm{C}-1, \mathrm{C}=\mathrm{O}), 92(\mathrm{~s}, \mathrm{C}-2,-\mathrm{CH}=), 176.8(\mathrm{~s}, \mathrm{C}-3), 124.1(\mathrm{~s}, \mathrm{C}-1$ ') $)$ 161.9(s,C2'), 118.8(s,C-3'), 136.0(s,C-4'), 127.6(s,C-5'), 132.0(s,C-6'), 128.6(t,C-1',,C-2', C6',), 132(d,C-3',,C-5'”), 121.6(s,C-4'’), UV/Vis(DMSO)nm: 360,410; EC-MS: 353.6 $(\mathrm{M}+23)$.

\section{Results and Discussion}

The 2-acetylphenyl benzoate 3(A-F) were prepared by the esterification of 2-hydroxy acetophenone $\mathbf{1}(\mathbf{A}-\mathbf{B})$ with aromatic carboxylic acids $\mathbf{2}(\mathbf{A}-\mathbf{C})$ in the presence of $\mathrm{POCl}_{3}$ (Scheme 1). 


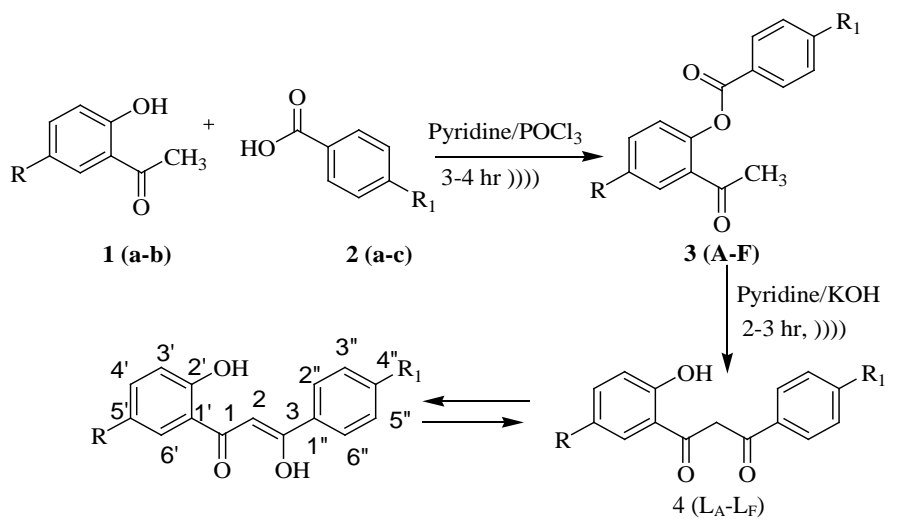

$4 \mathrm{~L}_{\mathrm{A}}: \mathrm{R}=\mathrm{H} ; \mathrm{R}_{1}=\mathrm{OCH}_{3}, 4 \mathrm{~L}_{\mathrm{B}}: \mathrm{R}=\mathrm{H} ; \mathrm{R}_{1}=\mathrm{OC}_{2} \mathrm{H}_{5}, 4 \mathrm{~L}_{\mathrm{C}}: \mathrm{R}=\mathrm{H} ; \mathrm{R}_{1}=\mathrm{Br}, 4 \mathrm{~L}_{\mathrm{D}}: \mathrm{R}=\mathrm{Cl} ; \mathrm{R}_{1}=\mathrm{OCH}_{3}, 4 \mathrm{~L}_{\mathrm{E}}: \mathrm{R}=$ $\mathrm{Cl} ; \mathrm{R}_{1}=\mathrm{OC}_{2} \mathrm{H}_{5}, 4 \mathrm{~L}_{\mathrm{F}}: \mathrm{R}=\mathrm{Cl} ; \mathrm{R}_{1}=\mathrm{Br}$

Scheme 1. Synthesis of ligands

1-(2-Hydroxyphenyl)-3phenylpropane-1,3-diones $\mathbf{4}\left(\mathbf{L}_{\mathbf{A}}-\mathbf{L}_{\mathbf{F}}\right)$ were prepared by BakerVenkatraman transformation ${ }^{21-22}$ of $\mathbf{3 ( A - F )}$ with $\mathrm{KOH}$ in pyridine. The ${ }^{1} \mathrm{H}$ NMR spectrum of $\mathbf{4} \mathbf{L}_{\mathbf{A}}$ exhibited a singlet at $\delta 15.9 \mathrm{ppm}$ due to enolic proton (since enol form in $\beta$-diketone is more stable), a singlet at $\delta 12.2 \mathrm{ppm}$ due to phenolic proton adjacent to the carbonyl group. ${ }^{13} \mathrm{C}$ NMR spectra gives singlet at $\delta 190.0 \mathrm{ppm}$ due to ketonic carbon C-1 and $\delta 185.1 \mathrm{ppm}$ due to enolic carbon C-3 confirming the keto-enol tautomerism in $\beta$-diketone $\mathbf{4} \mathbf{L}_{\mathrm{A}}$. The IR spectrum showed absorption bands at 2912.98(OH), 1708.01(C=O) and $1487.74 \mathrm{c} \mathrm{m}^{-1}(\mathrm{C}-\mathrm{O})$. The negative test for ester, the presence of characteristic ${ }^{1} \mathrm{H}$ NMR peaks and ${ }^{13} \mathrm{C}-\mathrm{NMR}$ peaks are consistent with the structure of 1-(2-hydroxyphenyl)-3-(4-methoxy phenyl) propane-1,3-dione $\mathbf{4} \mathbf{L}_{\mathbf{A}}$. The EC-MS spectrum showed a molecular ion peak at 270.28(M+23), confirms the molecular formula $\mathrm{C}_{16} \mathrm{H}_{14} \mathrm{O}_{4}$. UV/vis spectrum in DMSO generally showed intense peak in the region 360-412 nm confirms the $\alpha-\beta$ unsaturated carbonyl group of enol tautomerism indicating the presence of enolic structure ${ }^{23}$.

Comparative study results obtained by ultrasonication synthesis, versus conventional stirring method was that reaction which required 590 min by conventional method, was completed within 120 min by ultrasonication technique and yields have been improved from $70 \%$ to $85 \% \mathbf{4} \mathbf{L}_{\mathbf{A}}$. The comparison study data of ultrasonication and conventional method with physical data of the compounds are presented in Table 2.

Table 2. Physical data of substituted 1-(2-hydroxyphenyl)-3-phenyl propane-1,3-dione $4\left(L_{A}-L_{F}\right)$

\begin{tabular}{cccccc}
\hline & & \multicolumn{2}{c}{ With ultrasound $^{\mathrm{a}}$} & \multicolumn{2}{c}{ Without ultrasound $^{\mathrm{b}}$} \\
\cline { 3 - 6 } Compd. & M.P. ${ }^{0} \mathrm{C}$ & Time, min & Yield $^{\mathrm{c}}, \%$ & Time, min & Yield $^{\mathrm{c}}$ \% \\
\hline $\mathbf{4 L}_{\mathbf{A}}$ & $115-117$ & 120 & 85 & 590 & 70 \\
$\mathbf{4 L}_{\mathbf{B}}$ & $146-148$ & 120 & 85 & 590 & 73 \\
$\mathbf{4 L}_{\mathbf{C}}$ & $130-132$ & 110 & 87 & 570 & 75 \\
$\mathbf{4 L}_{\mathbf{D}}$ & $164-166$ & 120 & 82 & 600 & 74 \\
$\mathbf{4 L}_{\mathbf{E}}$ & $133-135$ & 120 & 84 & 590 & 72 \\
$\mathbf{4 L}_{\mathbf{F}}$ & $138-140$ & 110 & 88 & 570 & 71 \\
\hline
\end{tabular}

${ }^{a}$ Reaction of diketones under ultrasonic waves. ${ }^{b}$ Reaction of diketones under stir conditions. ${ }^{c}$ Isolated yield 
The reaction yield was improved with short time under sonication compared to that of conventional method ${ }^{24}$.

\section{Antimicrobial screening}

Antibacterial screening ${ }^{25}$ of cyclic $\beta$-diketones has been tested against one gram negative bacteria E.coli and two gram positive bacteria such as Staphylococcus aureus and Bacillus subtilis and antifungal screening ${ }^{26}$ has been tested against two species of fungi, Aspergillus niger and Fusarium Oxysporum by Kirby Baur's disc diffusion technique using dimethyl sulfoxide as a solvent. The streptomycin was used as reference in case of antibacterial and antifungal activity.

A uniform suspension of test organism of $24 \mathrm{~h}$. Old cultures was prepared in test tube containing sterile saline solution. A sterile nutrient agar was then added in each of the petri plates. The plates were rotated to ensure the uniform mixing of the micro organism in the agar medium which was then allowed to solidify. Sterile Whatmann filter paper disc were dipped in the solution of each compound and placed on the labeled plates. The DMSO was used a control of the solvent. The streptomycin was used as a standard compound for comparison. Plates were kept in refrigerator for half an hour for diffusion and then incubated at $37^{\circ} \mathrm{C}$ for $24 \mathrm{~h}$. After incubation the inhibitory zones around the discs were observed. The diameter on inhibition zones were measured in terms of $\mathrm{mm}$. Activity of each compound was compared with streptomycin as standard. The observed data of antimicrobial activity of compounds and the standard drugs are given in Table 3.

Table 3. Antimicrobial activity of compounds $4\left(\mathbf{L}_{\mathrm{A}}-\mathbf{L}_{\mathrm{F}}\right)$

\begin{tabular}{|c|c|c|c|c|c|c|}
\hline \multirow[b]{2}{*}{ Compd. } & \multirow[b]{2}{*}{$\begin{array}{c}\text { Conc., } \\
\text { ppm }\end{array}$} & \multicolumn{3}{|c|}{ Antibacterial activity } & \multicolumn{2}{|c|}{ Antifungal activity } \\
\hline & & $\begin{array}{l}\text { Bacillus } \\
\text { subtilis }\end{array}$ & E. coli & $\begin{array}{c}\text { Staphylococcus } \\
\text { aureus }\end{array}$ & $\begin{array}{l}\text { Aspergillus. } \\
\text { niger }\end{array}$ & $\begin{array}{l}\text { Fusarium. } \\
\text { oxysporum }\end{array}$ \\
\hline $4 \mathrm{~L}_{\mathrm{A}}$ & 100 & 8 & 10 & 7 & 8 & 11 \\
\hline $4 \mathrm{~L}_{B}$ & 100 & 11 & 8 & 8 & 7 & 8 \\
\hline $4 L_{C}$ & 100 & 11 & 11 & 7 & 12 & 12 \\
\hline $4 \mathrm{~L}_{\mathrm{D}}$ & 100 & 10 & 7 & 10 & 11 & 11 \\
\hline $4 L_{E}$ & 100 & 8 & 7 & 9 & 7 & 12 \\
\hline $4 \mathrm{~L}_{\mathrm{F}}$ & 100 & 10 & 9 & 9 & 7 & 13 \\
\hline Streptomycin & 100 & 6 & 7 & 6 & 6 & 6 \\
\hline
\end{tabular}

The screening results indicate the compounds $\mathbf{4}\left(\mathbf{L}_{\mathrm{A}}-\mathbf{L}_{\mathrm{F}}\right)$ showed moderate to excellent antimicrobial activities against the selected pathogens.

\section{Conclusion}

In the present work various1-(2-hydroxyphenyl)-3propane-1,3-diones $\mathbf{4}\left(\mathbf{L}_{\mathrm{A}}-\mathbf{L}_{\mathrm{F}}\right)$ were synthesiszed by Baker-Venkatraman transformation with $\mathrm{KOH}$ in pyridine by conventional as well as ultrasound irradiation and their structures confirmed on the basis of spectral analysis. ${ }^{1} \mathrm{H}$ NMR and ${ }^{13} \mathrm{C}$ NMR and UV/vis spectra revealed that the prepared compounds $\mathbf{4}\left(\mathbf{L}_{\mathrm{A}}-\mathbf{L}_{\mathrm{F}}\right)$ possess characteristic peaks due to presence of enolic proton (enol form of $\beta$ diketone) and phenolic proton adjacent to carbonyl group. These synthesized compounds were screened for in vitro antimicrobial activity and found to be promising candidate as new antibacterial as well as antifungal agents. In summary, this work demonstrates a rapid, efficient and environmentally friendly method for the synthesis of novel cyclic $\beta$-diketones $\mathbf{4}\left(\mathbf{L}_{\mathrm{A}}-\mathbf{L}_{\mathrm{F}}\right)$ under ultrasound irradiation and result obtained confirmed the superiority of ultrasound irradiation method over the conventional method. 


\section{Acknowledgment}

The authors are grateful to the Head, Dept. of Chemistry, Dr. Babasaheb Ambedkar Marathwada University, Aurangabad for providing the laboratory facility and Department of Chemistry, Pune University, Pune for providing spectral analysis of newly synthesized compounds and Dept. of Microbiology, Dayanand Science College, Latur for providing laboratory facility for carried out antimicrobial screening. Miss. Nanda Korde is highly thankful to UGC (WRO) Pune for providing Teacher Fellowship under FIP.

\section{References}

1. Taguchi Y, Sagara F, Kobayashi H and Ueno K, Bull Chem Soc Jpn., 1970, 43, 2470.

2. Siedle A., in comprehensive Coordination Chem.; Wilkinson, Pergamon Press, Oxford, 1987, Vol. 2 Chap 15.4, 365-412.

3. Tang L, Zhang S, Yang J, Gao W, Cui J and Zhuang T, Molecules, 2004, 9, 842-848.

4. Kumar R and Joshi C Y, Arkivoc, 2007, 9, 142.

5. Heller S and Natarajan S, Org Lett., 2006, 8, 2675-2678.

6. Simoni D, Invidiata F, Rondanin R, Grimaudo S, Cannizzo G, Barbusca E, Porretto F, Alessandro N and Tolomeo M, J Med Chem., 1999, 42, 4961.

7. Kuzueva O, Burgart Y, Saloutin V and Chupakhin O, Chemistry of Heterocyclic Compounds, 2001, 37, 1130.

8. $\quad$ Alekseev V V, Zelenin K N and Yakimovich S I, Russ J Org Chem., 1995, 31, 868.

9. Acton N, Brossi A, Newton D L and Sporn M B, J Med Chem., 1980, 23, 805-809.

10. Diana G D, Carabateas P M, Johnson R E, Williams G L, Pancic F and Collions J C, J Med Chem., 1978; 21(9), 889-894.

11. Bennett I, Broom N J, Cassels R, Elder J S, Masson N D and O’Hanlon P L, Bioorg Med Chem Lett., 1999, 9, 1847-1852.

12. Crouse G, McGowan M and Boisvenue R, J Med Chem., 1989, 32, 2148.

13. Nishiyama T, Shiotsu S and Tsujita H, Polym Degrad Stab., 2002, 76, 435.

14. Andrae I, Bringhen A, Bohm F, Gonzenbach H, Hill T, Mulroy L and Truscott T G, $J$ Photochem Photobiol B: Biol., 1997, 37, 147-150.

15. Dziemboska T and Rozwadowski Z, Curr Org Chem., 2001, 5, 289-313.

16. Tchertanov L and Mouscadet J F, J Med Chem., 2007, 50, 1133-1145

17. Singletary K, Macdonald C, Lovinelli M, Fisher C and Wallig M, Carcinogenesis, 1998, 19, 1039-1043

18. Lin C C, Wei G J, Huang M T and Ho C T, J Food Drug Anal., 2005, 13, 284

19. Lin C, Tsai Y, Huang M, Lu Y, Ho C, Tseng S and Teng S, Carcinogenesis, 2006, 27, 131-136

20. Rajgopal R, Jarikote D V and Srinivasan K V, Chem Commun., 2002, 616

21. Hauser C, Swamer F and Adama J, J Org React., 1954, 8, 168

22. Kraus C A, Fulton B S and Woo S H, J Org Chem., 1984, 49, 3212

23. Ahmed R, Malik M A and Haq M Z, J Chemical Soc Pak.,.1990, 12(4), 340.

24. Chate A V, Joshi R, Mandhane P and Gill C H, J Korean Cheml Soci., 2011, 55(4).

25. Sharma O P, Singla R K, Shrivastava B, Bhat V G, Shenoy G G, Jayashree B S and Sreenivasan K K, Indo Global J Pharm Sci., 2012, 2(1), 70-75.

26. Sharma O, Shrivastava B, Singla R K and Bhat V G, Indo Global JPharm Sci., 2011, 1(3), 252-257. 